\title{
Abordagem Empírica para Determinação da Permissividade Elétrica de Materiais Dielétricos Usando Cavidades Ressonantes
}

\author{
Vinícius Macedo Pereira, Gustavo Hardt, Marcos V. T. Heckler, Luis Enrique Gomez Armas
}

\begin{abstract}
Resumo-Este artigo apresenta um estudo sobre a caracterização da constante dielétrica $\left(\varepsilon_{r}\right)$ de materiais pelo método da cavidade ressonante. Analisa-se o comportamento da frequência de ressonância $\left(f_{c}\right)$ em função da variação do material e da espessura das amostras. Amostras de dois materiais diferentes, PEAD e Nylon, com três espessuras diferentes $(3,2 \mathrm{~mm}, 4,4 \mathrm{~mm}$ e $5,5 \mathrm{~mm}$ ) foram consideradas neste estudo. Observou-se que as amostras com espessuras de $4,4 \mathrm{~mm}$ e $5,5 \mathrm{~mm}$ propiciam leituras mais precisas de $f_{c}$, e, consequentemente, melhores estimativas para $\varepsilon_{r}$. Verificou-se, também, que as equações de calibração propostas são ferramentas satisfatórias para o cálculo de $\varepsilon_{r}$ a partir de medidas de parâmetros $S$ com auxílio de um analisador vetorial de sinais.
\end{abstract}

Palavras-Chave-Cavidade Ressonante, Frequência de Ressonância, Constante Dielétrica.

Abstract-This paper presents a study about the dielectric constant $\left(\varepsilon_{r}\right)$ characterization of different materials with the resonant cavity method. The analysis of the variation of the resonance frequency $\left(f_{c}\right)$ has been carried out for two different factors: material and thickness of samples. Two different materials, PEAD and Nylon, and three different thicknesses $(3.2 \mathrm{~mm}$, $4.4 \mathrm{~mm}$ e $5.5 \mathrm{~mm}$ ) have been analyzed. The results indicate that higher precision in the determination of $f_{c}$ and $\varepsilon_{r}$ can be obtained for the samples with thicknesses of $4.4 \mathrm{~mm}$ and $5.5 \mathrm{~mm}$. Experimental data collected with measurements of $S$-parameters carried out with a vector network analyzer allowed to validate the proposed equations and callibration approach to assess $\varepsilon_{r}$.

Keywords-Resonant Cavity, Resonance Frequency, Dielectric Constant.

\section{INTRODUÇÃO}

As propriedades dielétricas dos materiais são de particular interesse em Telecomunicações, principalmente em teoria de linhas de transmissão e eletromagnetismo. $\mathrm{Na}$ indústria de dispositivos de comunicação, dielétricos são empregados na fabricação de alimentadores baseados em lentes eletromagnéticas para antenas refletoras. Outro exemplo é a fabricação de circuitos impressos, que necessitam de laminados com

Vinícius Macedo Pereira, Grupo de Óptica, Micro e Nanofabricação de Dispositivos - GOMNDI, Universidade Federal do Pampa - Unipampa, Alegrete-RS, e-mail: viniciusmp2.aluno@unipampa.edu.br; Gustavo Hardt Grupo de Óptica, Micro e Nanofabricação de Dispositivos - GOMNDI, Universidade Federal do Pampa - Unipampa, Alegrete-RS, e-mail: gustavohardt35@gmail.com; Marcos Vinício Thomas Heckler, Laboratório de Eletromagnetismo, Micro-ondas e Antenas - LEMA, Universidade Federal do Pampa - Unipampa, Alegrete-RS, e-mail: marcosheckler@unipampa.edu.br Luis Enrique Gomez Armas, Grupo de Óptica, Micro e Nanofabricação de Dispositivos - GOMNDI, Universidade Federal do Pampa - Unipampa, Alegrete-RS, e-mail: luisarmas@unipampa.edu.br. Este trabalho foi financiado por bolsa de pesquisa nível mestrado da CAPES. baixas perdas em micro-ondas e elevado controle de suas propriedades físicas e elétricas.

As principais propriedades dielétricas são a permissividade elétrica relativa $\left(\varepsilon_{r}\right)$ e a tangente de perdas $(\tan \delta)$ [1], [2]. A permissividade elétrica consiste em um parâmetro constitutivo dos materiais que relaciona a capacidade de reforço da densidade de fluxo elétrico sob a aplicação de campo elétrico externo, através da polarização das cargas elétricas ou íons no material. Esta propriedade é medida em Farads por metro (F/m) [1], [4].

Considerando o espaço livre, tem-se a permissividade elétrica do vácuo $\left(\varepsilon_{0}\right)$, que equivale a $10^{-9} /(36 \pi) \mathrm{F} / \mathrm{m}$. Esta grandeza pode ser vista como um valor de referência para as constantes de permissividade elétrica dos materiais dielétricos, visto que todos possuem $\varepsilon$ superior a $\varepsilon_{0}$, devido à ausência de cargas elétricas e íons no vácuo e, consequentemente, à ausência do fenômeno de polarização elétrica. Para fins práticos, é comum definir-se um valor normalizado de permissividade elétrica, tomando-se como fator de normalização $\varepsilon_{0}$. Esta grandeza normalizada é denominada permissividade elétrica relativa ou constante dielétrica, sendo, matematicamente, dada por [1], [4],

$$
\varepsilon_{r}=\frac{\varepsilon}{\varepsilon_{0}} .
$$

Um método clássico utilizado para determinação de $\varepsilon_{r}$ é a técnica da cavidade ressonante, que consiste em um dispositivo cilíndrico ou retangular metálico, oco e hermético, preenchido por ar. Ao se inserir uma amostra dielétrica com $\varepsilon_{r}>1$, a frequência de ressonância $\left(f_{c}\right)$ tende a diminuir, devido à perturbação no campo eletromagnético no interior da cavidade. A partir desta alteração em $f_{c}$, extrai-se a informação referente à constante $\varepsilon_{r}$ da amostra em análise [3], [5], [7].

Rubinger e Costa [6] apresentaram um estudo sobre a influência da variação de volume de amostras dielétricas sobre o comportamento de $f_{c}$ de uma cavidade ressonante. Matematicamente, os autores relacionaram, através de uma equação linear, a constante $\varepsilon_{r}$ e o volume das amostras com a razão $\left(f_{c}-f_{a}\right) / f_{c}$, onde $f_{a}$ representa a frequência de ressonância da cavidade com uma amostra inserida. Os materiais analisados foram os polímeros polianilina (PANI) e teflon (PTFE).

Liu et al. [7] apresentaram um estudo utilizando a cavidade ressonante cilíndrica para a determinação da composição química de compostos formados sob catalisadores de ácido zeólito. Esta informação foi obtida pela estimativa da constante de perdas das amostras, que é dependente da parte complexa de $\varepsilon$ $\left(\varepsilon^{\prime \prime}\right)$, através da variação de $f_{c}$. Kombolias et al. [8] utilizaram 
o princípio da cavidade ressonante com perturbação material como método-base para o desenvolvimento de uma técnica de caracterização de materiais não destrutiva, para a investigação da matéria-prima precursora de diferentes amostras de papel.

Neste artigo, um estudo teórico e experimental foi realizado para verificar a influência da espessura das amostras na precisão da leitura das mudanças da frequência de ressonância de uma cavidade cilíndrica. A seguinte seção apresenta a metodologia adotada para o desenvolvimento do procedimento. Na seção III, os resultados obtidos a partir de simulações eletromagnéticas são apresentados. A validação com os resultados experimentais é apresentada na seção IV. Por fim, apresentam-se as conclusões do trabalho.

\section{Metodologia}

Neste estudo, considerou-se a cavidade com geometria cilíndrica. A frequência central foi escolhida como $3,5 \mathrm{GHz}$, de acordo com o padrão para testes de materiais e dispositivos em desenvolvimento visando a atender a tecnologia de quinta geração de sistemas de comunicações móveis (5G) [9], [10].

As dimensões físicas da cavidade podem ser calculadas a partir de algum dos primeiros modos de ressonância: $\mathrm{TE}_{111}$ ou $\mathrm{TM}_{010}$ [4], [11]. Assim, para o modo TE, e considerando que seu interior seja preenchido por ar, $f_{c}$ se relaciona com as dimensões internas da cavidade cilíndrica por

$$
\left(f_{c}\right)_{m n p}(T E)=\frac{1}{2 \pi \sqrt{\mu_{0} \varepsilon_{0}}} \sqrt{\left(\frac{\chi_{m n}^{\prime}}{a}\right)^{2}+\left(\frac{p \pi}{d}\right)^{2}},
$$

onde $a$ é o raio interno, $d$ é a altura interna, os parâmetros $m, n$ e $p$ são índices correspondentes ao modo de operação, e $\chi_{m n}^{\prime}$ representa o $n$-ésimo zero da derivada da função de Bessel de primeira espécie $J_{m}(x)$, cujos valores tabelados estão disponíveis em [12].

A estimativa inicial das dimensões físicas da cavidade deuse a partir do primeiro modo de ressonância $\left(\mathrm{TE}_{111}\right)$. Fixou-se também, previamente, que $d=65 \mathrm{~mm}$. Assim, manipulandose a equação (2) de modo a deixar o raio $a$ em função das demais variáveis conhecidas, e sendo $\chi_{11}^{\prime}=1,8412$ [12], temse que

$$
a=\frac{1,8412}{\sqrt{\left(2 \pi 3,5 \times 10^{9} \sqrt{\mu_{0} \varepsilon_{0}}\right)^{2}-\left(\frac{\pi}{65 \times 10^{-3}}\right)^{2}}}=33,380 \mathrm{~mm} .
$$

Considerando os valores calculados, utilizou-se o software HFSS $^{\circledR}$ para verificação da operação da cavidade projetada. O modelo elaborado é apresentado na Figura 1. Além da cavidade cilíndrica, dois conectores SMA, juntamente com dois excitadores, foram adicionados à estrutura. Optou-se por tais conectores, por apresentarem menores perdas eletromagnéticas na frequência de projeto.

$\mathrm{Na}$ extremidade oposta às fontes de excitação, um rebaixo com diâmetro de $30 \mathrm{~mm}$ e profundidade de $1 \mathrm{~mm}$ foi introduzido, para garantir o correto encaixe das amostras em seu interior. Os monopolos foram dimensionados, inicialmente, com comprimento próximo a $\lambda_{0} / 4$, ou, aproximadamente,

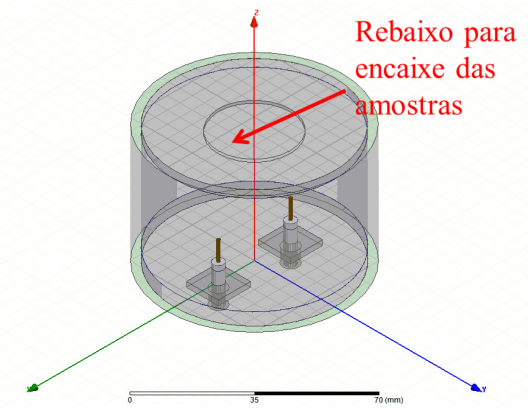

Fig. 1. Modelo de simulação eletromagnética da cavidade cilíndrica.

$20 \mathrm{~mm}$. A distância entre os dois pinos foi ajustada de modo a obter-se um acoplamento de energia satisfatório entre as portas.

\section{Resultados Simulados}

A Tabela III mostra as dimensões físicas da cavidade, estimadas inicialmente e ajustadas nas simulações eletromagnéticas no HFSS ${ }^{\circledR}$. Pode-se observar que existe uma considerável diferença entre o parâmetro $d$ estimado inicialmente e o obtido nas simulações. Estas discrepâncias devem-se, principalmente, à excitação da cavidade ressonante e ao rebaixo inserido, pois estes fatores não são devidamente modelados na equação (2). Esta mesma explicação aplica-se à diferença entre os comprimentos dos pinos de excitação, cujo valor obtido após as simulações foi de $7,560 \mathrm{~mm}$. Para melhorar o acoplamento de energia entre os dois monopolos, estes foram afastados entre si de $12,500 \mathrm{~mm}$.

TABELA I

DiMENSÕES FÍsicAS DA CAVIDADE RESSONANTE.

\begin{tabular}{|c||c|c|}
\hline Dimensão & Valor estimado & Valor simulado \\
\hline$a$ & $33,380 \mathrm{~mm}$ & $32,250 \mathrm{~mm}$ \\
\hline$d$ & $65,000 \mathrm{~mm}$ & $41,500 \mathrm{~mm}$ \\
\hline$l$ & $20,000 \mathrm{~mm}$ & $7,560 \mathrm{~mm}$ \\
\hline
\end{tabular}

Na Figura 2, pode-se observar que a máxima concentração de intensidade de campo elétrico no interior da estrutura ocorre em sua região central e é praticamente nula nas paredes laterais do cilindro.

A Figura 3 mostra curvas dos parâmetros $S_{11}$ e $S_{21}$ simulados. O nível do coeficiente de reflexão é considerado aceitável $\left(S_{11}<-15 \mathrm{~dB}\right.$, ou seja, apenas $3,1 \%$ da energia aplicada às portas da cavidade é refletida à fonte de excitação) e devidamente sintonizado na frequência de projeto, $f_{c}=$ 3,5 GHz.

Para as amostras analisadas, considerou-se a geometria cilíndrica (para um melhor encaixe e centralização no rebaixo), com diâmetro de $28 \mathrm{~mm}$ e espessura $(h)$ variando entre $3,2 \mathrm{~mm}, 4,4 \mathrm{~mm}$ e $5,5 \mathrm{~mm}$ para dois materiais distintos $\left(\varepsilon_{r}=3,1\right.$ para o Nylon e $\varepsilon_{r}=2,25$ para o PEAD). Sua representação tridimensional é mostrada na Figura 4. Assim, realizaram-se simulações da variação de $f_{c}$ com a inserção de amostras de diferentes espessuras e materiais. Para cada combinação de espessura e material, observaram-se 4 


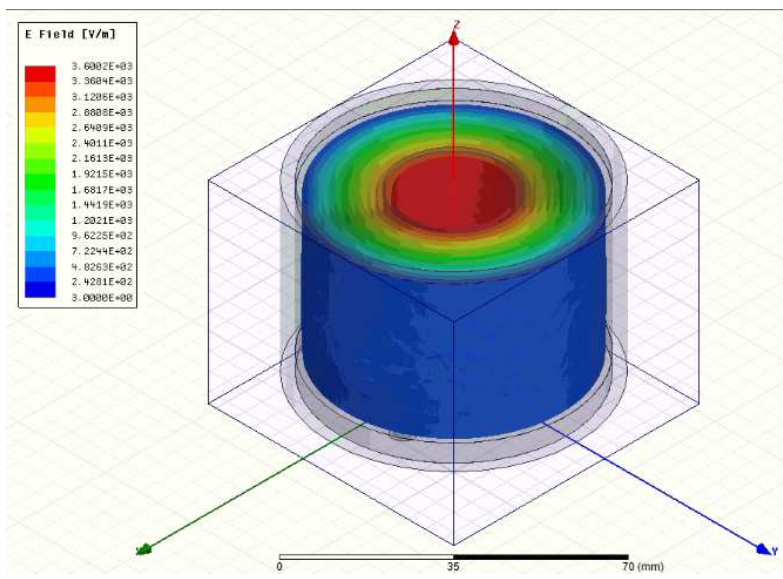

Fig. 2. Distribuição de campo elétrico no interior da cavidade.

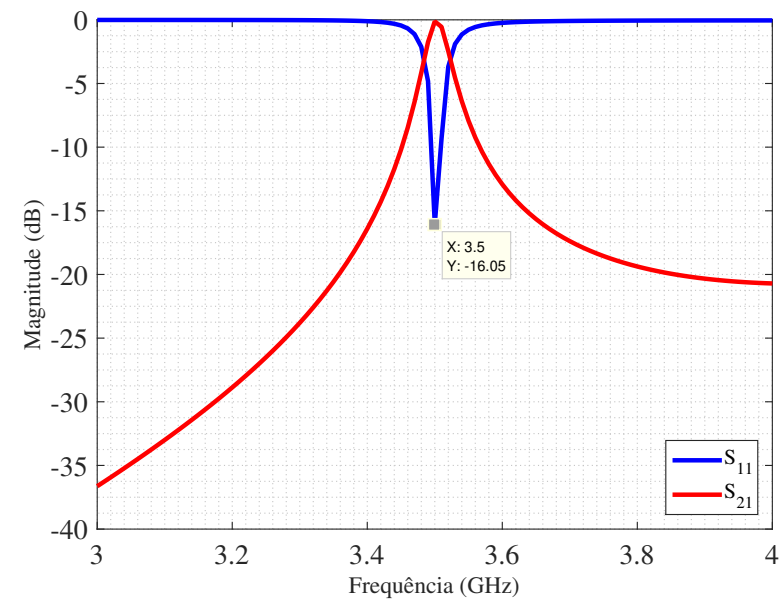

Fig. 3. Parâmetros $S$ simulados considerando a cavidade vazia.

amostras com $\varepsilon_{r}$ variando dentro de seu nível de tolerância típico. Nestas simulações, aplicaram-se, então, os conceitos de análise de experimentos fatoriais, descritos em [13]. Com isto, obtiveram-se os termos de soma dos quadrados (SQ), graus de liberdade (GL), média quadrática (MQ) e $F_{0}$ calculado (relacionado ao teste de hipótese nula - $H_{0}$ ), relacionados ao fator A (material da amostra) e fator B (espessura da amostra). $\mathrm{Na}$ Tabela II, mostra-se a análise fatorial deste experimento.

TABELA II

ANÁLISE ESTATÍSTICA FATORIAL DO EXPERIMENTO COMPUTACIONAL.

\begin{tabular}{|c||c|c|c|c|}
\hline Parâmetro & $S Q$ & $G L$ & $M Q$ & $F_{0}$ \\
\hline Material & $2,185 \times 10^{-3}$ & 1 & $2,185 \times 10^{-3}$ & 552,011 \\
\hline Espessura & $8,100 \times 10^{-3}$ & 2 & $4,050 \times 10^{-3}$ & 1023,158 \\
\hline Interação & $0,196 \times 10^{-3}$ & 2 & $0,098 \times 10^{-3}$ & 24,800 \\
\hline Erro & $0,071 \times 10^{-3}$ & 18 & $0,004 \times 10^{-3}$ & \\
\hline Total & $10,553 \times 10^{-3}$ & 23 & & \\
\hline
\end{tabular}

Considerando-se um nível de significância $\alpha=0,01$, e utilizando-se a tabela $F$ de distribuições de probabilidades em [14], obtiveram-se os valores tabelados de $F_{0}$. Na Tabela III, demonstram-se, de forma comparativa, os valores de $F_{0}$ calculados e tabelados.

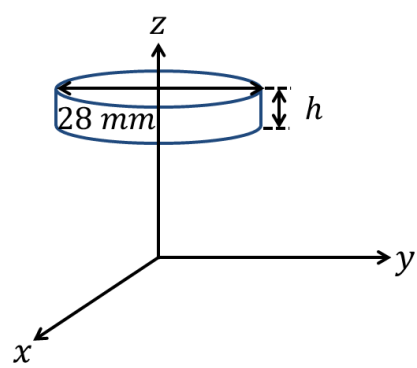

Fig. 4. Representação tridimensional da geometria das amostras.

TABELA III

ANÁLISE ESTATÍSTICA FATORIAL DO EXPERIMENTO COMPUTACIONAL.

\begin{tabular}{|c||c|c|}
\hline Parâmetro & $F_{0}$ calculado & $F_{0}$ tabelado \\
\hline Material & 552,011 & 8,285 \\
\hline Espessura & 1023,158 & 6,013 \\
\hline Interação & 24,800 & 6,013 \\
\hline
\end{tabular}

Pode-se observar, pela comparação entre os parâmetros $F_{0}$ calculados e tabelados, que, considerando o efeito do fator $A$, a hipótese nula $H_{0}: \tau_{i}=0$ é falsa, pois $F_{0}$ (calculado) $=$ $552,011>F_{0}$ (tabelado $)=8,285$. Ou seja, a variação de $f_{c}$ é dependente do material da amostra. Assim, diferentes tipos de materiais podem corresponder a diferentes constantes $\varepsilon_{r}$, implicando em diferentes deslocamentos de $f_{c}$. No caso do fator $B$, observa-se, também, que a hipótese nula $H_{0}: \tau_{i}=0$ é falsa, pois $F_{0}($ calculado $)=1023,158>F_{0}($ tabelado $)=$ 6,013 . Assim, interpreta-se que a variação de $f_{c}$ é dependente, também, da espessura da amostra, pois, diferentes espessuras implicam em diferentes volumes de material dielétrico no interior da cavidade que, por sua vez, propiciam diferentes deslocamentos de $f_{c}$.

Para complementar a análise estatística, mostra-se, na Figura 5 , o gráfico de caixas para as médias de $f_{c}$ observadas no experimento. Constata-se que, utilizando-se amostras com espessura de $4,4 \mathrm{~mm}$ ou $5,5 \mathrm{~mm}$, a variação na resposta de $f_{c}$, considerando diferentes materiais, é mais visível e, consequentemente, possibilita maior precisão para o cálculo de $\varepsilon_{r}$.

\section{RESUlTAdOS EXPERIMENTAIS}

Para validação prática do estudo desenvolvido, construiu-se um protótipo da cavidade cilíndrica (ver Figura 6) e efetuaramse medições de seus parâmetros $S$. Na Figura 7, mostrase comparação entre os parâmetros $S$ simulados e medidos, considerando a cavidade vazia.

Como visto na Figura 7, o protótipo construído apresentou $f_{c}=3,49 \mathrm{GHz}$, que é ligeiramente inferior à frequência central de projeto $(3,5 \mathrm{GHz})$. Esta pequena diferença pode ser dada pela imprecisão intrínseca ao processo construtivo da cavidade cilíndrica. Porém, ainda assim, a ressonância observada está satisfatoriamente próxima à frequência inicial do projeto.

Em sequência, prepararam-se amostras de diâmetro $28 \mathrm{~mm}$ e com espessuras de $h=3,2 \mathrm{~mm}, h=4,4 \mathrm{~mm}$ e $h=5,5 \mathrm{~mm}$ dos materiais PEAD e Nylon, que foram inseridas, uma por 


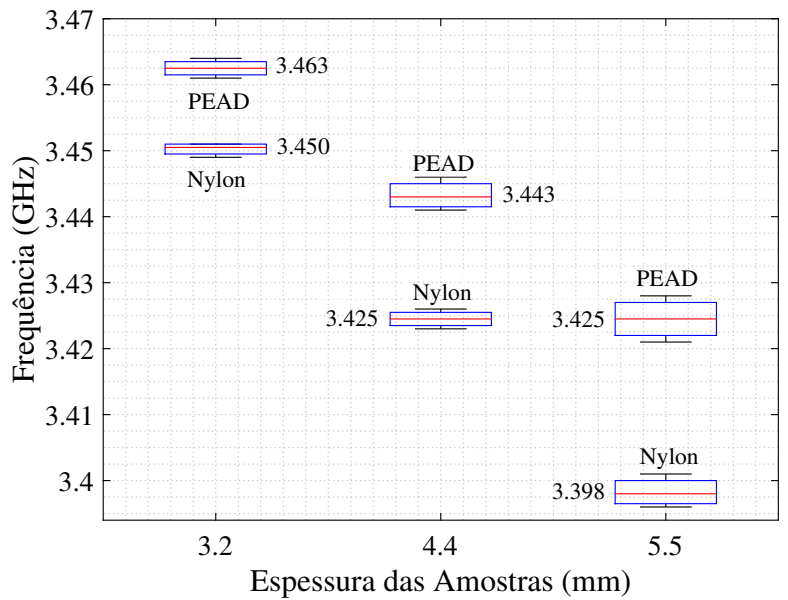

Fig. 5. Análise por gráfico de caixas da variação da frequência da cavidade pela variação de material e espessura das amostras.

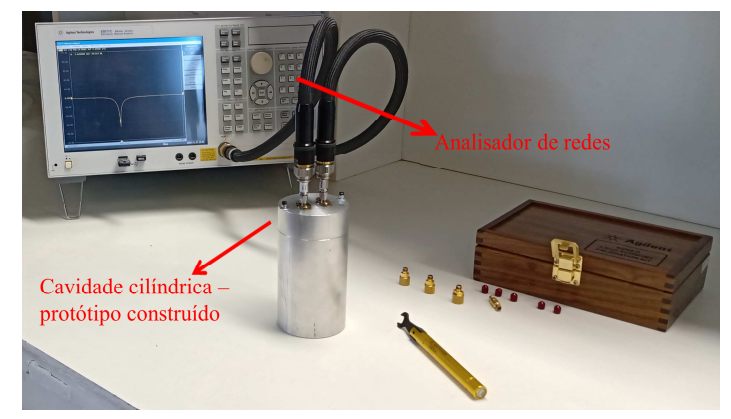

Fig. 6. Setup de medição e protótipo construído.

vez, no interior do protótipo. Os parâmetros $S_{11}$ medidos para as amostras de PEAD e de Nylon são mostrados nas Figuras 8 e 9, respectivamente. As frequências de ressonância medidas para cada amostra são aproximadamente semelhantes às frequências médias observadas em simulação (Figura 5), visto que a diferença de $10 \mathrm{MHz}$ vista na comparação entre os resultados simulados ( $\mathrm{sim}$ ) e medidos (med) para a cavidade vazia foi, também, observada na comparação entre os resultados simulados e medidos considerando as amostras (Tabela IV).

TABELA IV

COMPARAÇÃO ENTRE VALORES DE $f_{c}$ SIMULAdOS E MEDIDOS.

\begin{tabular}{|c||c|c|c|c|}
\hline \multicolumn{1}{|c||}{} & \multicolumn{2}{c|}{$f_{c}(\mathrm{GHz})$} & $-\mathrm{PEAD}$ & \multicolumn{2}{c|}{$f_{c}(\mathrm{GHz})$} & - Nylon \\
\hline$h(\mathrm{~mm})$ & $($ sim $)$ & $($ med $)$ & $($ sim $)$ & $($ med $)$ \\
\hline 3,2 & 3,461 & 3,450 & 3,450 & 3,440 \\
\hline 4,4 & 3,443 & 3,430 & 3,425 & 3,415 \\
\hline 5,5 & 3,425 & 3,410 & 3,398 & 3,390 \\
\hline
\end{tabular}

Como etapa final, desenvolveu-se uma relação matemática entre a razão $\left(f_{c}-f_{a}\right) / f_{c}$ e $\varepsilon_{r}$ a ser calculado, tomando como base o estudo proposto em Rubinger e Costa [6] e ajustando-se o formalismo para a geometria de cavidade e amostras deste estudo. O modelo de simulação no $\mathrm{HFSS}^{\circledR}$ foi modificado, de modo que $f_{c}=3,49 \mathrm{GHz}$ e, posteriormente, efetuaramse simulações eletromagnéticas variando $\varepsilon_{r}$ da amostra de 2

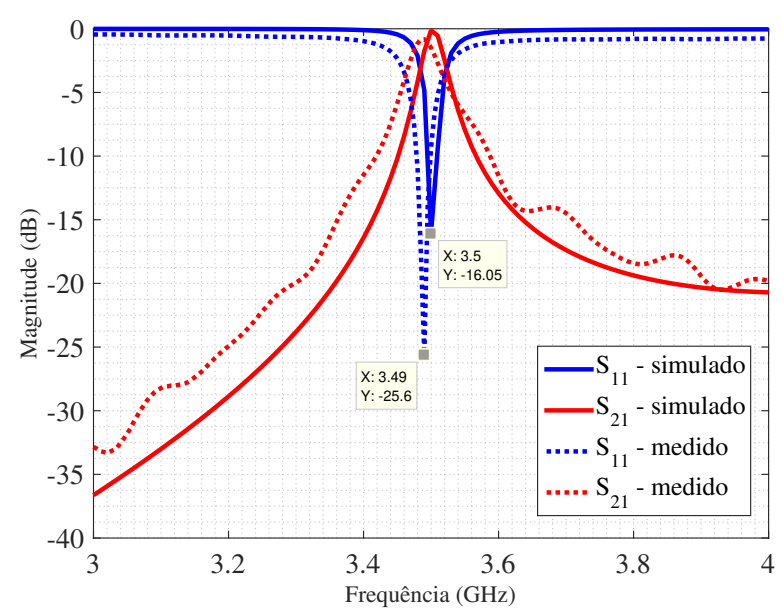

Fig. 7. Comparação entre parâmetros $S$ simulados e medidos - cavidade vazia.

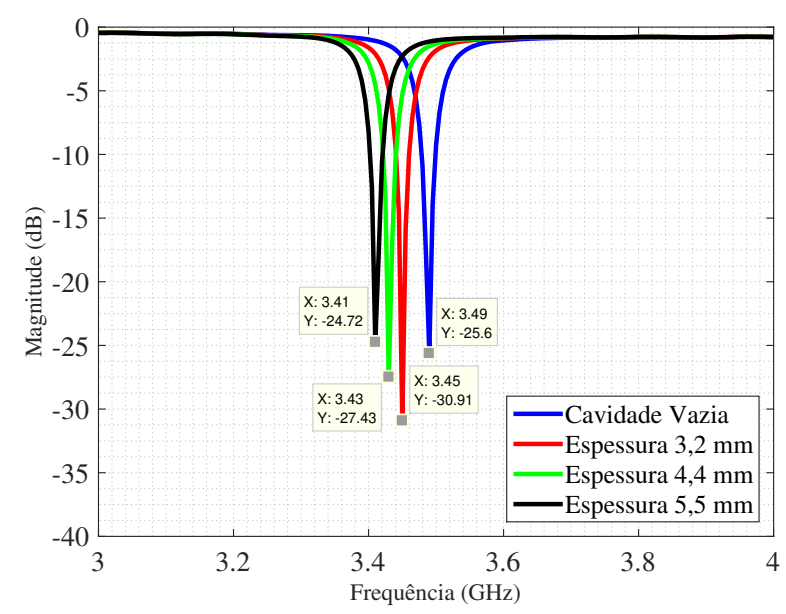

Fig. 8. Parâmetros $S_{11}$ medidos - amostras PEAD.

até 20 com passo de 1 para cada uma das três espessuras analisadas. Os resultados obtidos foram utilizados para plotar as três curvas de calibração, vistas na Figura 10.

Observa-se que, diferentemente de Rubinger e Costa [6], onde seu experimento foi descrito matematicamente por meio de uma relação linear, o presente experimento, por sua vez, é satisfatoriamente modelado matematicamente através de equações logarítmicas. Com o software Excel ${ }^{\circledR}$, estimaramse as equações que modelam cada curva da Figura 10. Assim, para $h=3,2 \mathrm{~mm}$,

$$
\frac{f_{c}-f_{a}}{f_{c}}=0,0051 \ln \left(\varepsilon_{r}-1\right)+0,0104
$$

para $h=4,4 \mathrm{~mm}$,

$$
\frac{f_{c}-f_{a}}{f_{c}}=0,0094 \ln \left(\varepsilon_{r}-1\right)+0,0145 ;
$$

para $h=5,5 \mathrm{~mm}$,

$$
\frac{f_{c}-f_{a}}{f_{c}}=0,0155 \ln \left(\varepsilon_{r}-1\right)+0,0174 .
$$




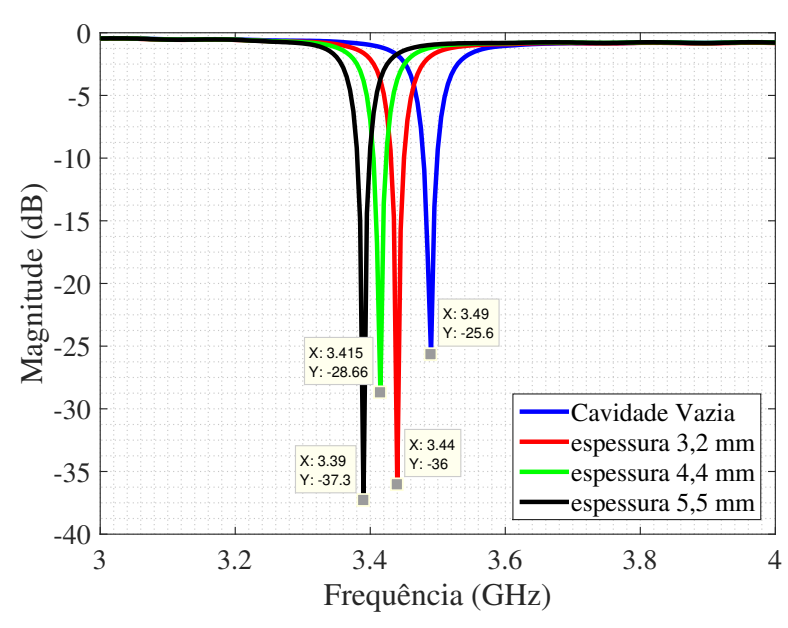

Fig. 9. Parâmetros $S_{11}$ medidos - amostras Nylon.

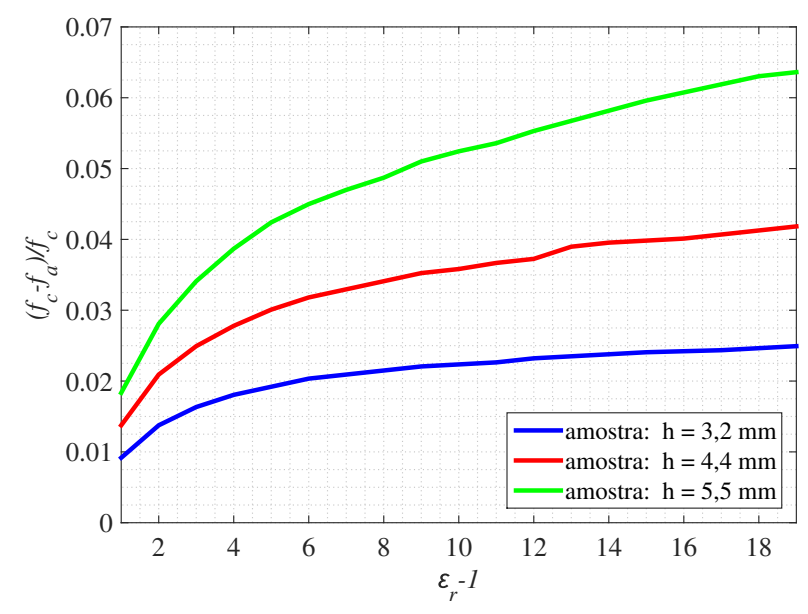

Fig. 10. Curvas de calibração para o cálculo de $\varepsilon_{r}$ a partir de $f_{c}$ medido.

Para o cálculo de $\varepsilon_{r}$ de cada amostra (de acordo com sua espessura), manipularam-se as equações (4) a (6), de modo a deixar o termo $\varepsilon_{r}$ em evidência no lado esquerdo da igualdade. Como resultado, a tabela $\mathrm{V}$ mostra os resultados de $\varepsilon_{r}$ calculados para cada amostra medida.

\section{TABELA V}

CONSTANTES $\varepsilon_{r}$ CALCULADAS PARA AS AMOSTRAS ANALISADAS.

\begin{tabular}{|c|c|c|c|c|}
\hline & PEAD & PEAD & Nylon & Nylon \\
\hline$h(\mathrm{~mm})$ & $f_{a}(\mathrm{GHz})$ & $\varepsilon_{r}$ & $f_{a}(\mathrm{GHz})$ & $\varepsilon_{r}$ \\
\hline 3,2 & 3,450 & 2,231 & 3,440 & 3,160 \\
\hline 4,4 & 3,430 & 2,332 & 3,415 & 3,104 \\
\hline 5,5 & 3,410 & 2,430 & 3,390 & 3,067 \\
\hline
\end{tabular}

Os resultados obtidos apresentaram considerável fidedignidade àqueles comercialmente especificados para os materiais analisados. Observou-se, para o caso do PEAD, que houve uma pequena variação entre os valores calculados para cada espessura de amostra, a qual pode ser considerada como dentro dos limites de tolerância que geralmente são especificados pelos fabricantes. Porém, para as amostras de Nylon, pôdese constatar que, para todas as amostras medidas, a constante $\varepsilon_{r}$ calculada resultou em valores substancialmente próximos a 3,1 , que é o seu valor especificado comercialmente.

\section{CONCLUSÃO}

$\mathrm{O}$ presente trabalho apresentou a caracterização teórica e experimental do método da cavidade ressonante, dimensionado para que $f_{c}=3,5 \mathrm{GHz}$ (padrão $5 \mathrm{G}$ ). A validação de seu funcionamento possibilita sua aplicação, em sequência, na caracterização de materiais dielétricos alternativos baseados em matéria-prima oriunda de resíduos vegetais, tais como sílica proveniente da casca de arroz e do capim annoni. Observou-se que as espessuras de amostra mais adequadas são de 4,4 mm e $5,5 \mathrm{~mm}$, dada a mais apurada precisão nas leituras da variação de $f_{c}$. Também constatou-se que as equações propostas para cada espessura de amostra do experimento são confiáveis para o cálculo de $\varepsilon_{r}$ de materiais cujas propriedades dielétricas ainda não sejam totalmente conhecidas.

\section{AGRADECIMENTOS}

Os autores agradecem à Universidade Federal do Pampa (Unipampa) pela estrutura laboratorial oferecida e à Coordenação de Aperfeiçoamento de Pessoal de Nível Superior (CAPES) pelo auxílio financeiro por meio de bolsa de pesquisa em nível de mestrado.

\section{REFERÊNCIAS}

[1] W. D. Callister. Ciência e Engenharia de Materiais. Uma Introdução. 7.ed. LTC, 2008

[2] C. Bowick. RF Circuit Design. [S.I.]: Newnes, 2011

[3] C. W. Sayre. Complete Wireless Design. 2.ed. Mc Graw Hill, 2008.

[4] C. A. Balanis. Advanced Engineering Electromagnetics. John Wiley \& Sons, 1989.

[5] Z. Li and E. Li. "Study on Measurement of Dielectric Constant of Partially Filled Material using Modified Cavity Perturbation Technique," in The 2012 International Workshop on Microwave and Millimeter Wave Circuits and System Technology, Chengdu, China, Abr. 2012. DOI: 10.1109/MMWCST.2012.6238166.

[6] C. P. L. Rubinger and L. C. Costa. "Building a Resonant Cavity for the Measurement of Microwave Dielectric Permittivity of High Loss Materials," in Microwave and Optical Technology Letters, Jul. 2007, pp. 1687-1690. DOI: 10.1002/mop.

[7] B. Liu, D. R. Slocombe, J. Wang, A. Aldawsari, S. Gonzalez-Cortes, J. Arden, V. L. Kuznetsov, H. Al Megren, M. Al Kinany, T. Xiao and P. P. Edwards. "Microwaves Effectively Examine the Extent and Type of Coking over Acid Zeolite Catalysts," in Nature Communications, Set. 2017. DOI: 10.1038/s41467-017-00602-8.

[8] M. Kombolias, J. Obrzut, M. Postek, D. Poster and Y. S. Obeng. "Contactless Resonant Cavity Dielectric Spectroscopic Studies of Cellulosic Paper Aging," in Analytical Letters, Ago. 2019. DOI: 10.1080/00032719.2019.1655648.

[9] ANATEL, Agência Nacional de Telecomunicações. Frequências Destinadas ao $5 G$ têm Novo Ato e Consulta sobre Requisitos Técnicos. Brasília, DF, 2020. Disponível em $<$ https://www.anatel.gov.br/institucional/mais-noticias/2610frequencias-destinadas-ao-5g-tem-novo-ato-e-consulta-sobre-requisitostecnicos >. Acesso em: 09 mar. 2021.

[10] ETSI, European Telecommunications Standards Institute Technologies: Mobile - 5G. 2020.. Disponível em $<$ https://www.etsi.org/technologies/mobile/5g $>$. Acesso em: 09 mar. 2021.

[11] R. E. Collin. Foundations for Microwave Engineering. 2.ed. IEEE Press. John Wiley \& Sons, 2001.

[12] D. M. Pozar. Microwave Engineering. 4.ed. John Wiley \& Sons, 2011.

[13] D. C. Montgomery e G. C. Runger. Estatística Aplicada e Probabilidade para Engenheiros. 6.ed. LTC, 2016.

[14] E. A. Reis. Tabela de Limites Unilaterais da Distribuição F de Fisher. Disponível em <http://www.est.ufmg.br/ edna/Tabela-F.pdf $>$. Acesso em: 09 mar. 2021. 